\title{
Association between Aphasia and Acalculia: Analytical Cross-Sectional Study
}

\author{
Gabriela De Luccia', Karin Zazo Ortiz ${ }^{2}$ \\ ${ }^{1}$ Department of Speech Pathology, Federal University of São Paulo (UNIFESP), São Paulo, Brazil \\ ${ }^{2}$ University Center of Várzea Grande, Mato Grosso, Brazil \\ Email: gabideluccia@hotmail.com
}

Received 22 September 2015; accepted 11 January 2016; published 14 January 2016

Copyright (C) 2016 by authors and Scientific Research Publishing Inc.

This work is licensed under the Creative Commons Attribution International License (CC BY). http://creativecommons.org/licenses/by/4.0/

(c) (i) Open Access

\begin{abstract}
Acalculia in aphasic patients should be better investigated in order to understand if it is a simple comorbid or if it is influenced by language disorders. This study aimed to compare the performance on EC301 battery calculation tasks between aphasic and normal subjects and sought to verify a possible association between number processing and calculation skills and linguistic changes in aphasic patients, in order to investigate if language disorders interfere with number processing and calculation. Analytical cross-sectional study with a control group, performed of the Department of Speech and Hearing Disorders of a public university, conducted in the city of São Paulo, Brazil. First, to analyze the specific difficulties encountered in numerical processing and calculation tasks among the aphasic group, aphasic and healthy adult's performance in specific calculation tasks were compared. The calculation tasks, which had been badly performed by aphasic patients, were selected. Aphasic patients were also submitted to the language tasks from Montreal-Toulouse Protocol: oral and written comprehension, repetition, reading aloud, naming and dictation. We observed that aphasic individuals showed changes in numerical processing and calculation tasks that were not observed in the healthy population. The most important finding of this study was that aphasic individuals showed changes in numerical processing and calculation that were positively associated to their linguistic performance. The strong associations between battery EC301 and linguistic tasks suggest that language disorders interfere with number processing and calculation.
\end{abstract}

\section{Keywords}

Dyscalculia, Aphasia, Stroke, Disabled Persons, Language 


\section{Introduction}

Neurological and neuropsychological clinical reports frequently mention acalculia, but specific analyses of acalculia are rather limited. Calculation ability represents an extremely complex cognitive process, which requires multifactorial processes, including verbal, spatial, memory, and executive functions [1] [2]. According to Ardila, Rosselli (2002) aphasia is an impairment of language, affecting the production or comprehension of speech and the ability to read or write. Aphasia is always due to injury on the brain-most commonly from a stroke, particularly in older individuals. On the other hand, acalculia as a complete inability or part of dealing with numbers, and these authors, represents a acalculia commitment in numerical processing and calculation that may result from a brain injury such as strokes, cerebral traumas, dementias and degenerative diseases.

Acalculia following brain injury is not uncommon. Therefore, acalculia in aphasic patients should be better investigated in order to understand if it is a simple comorbid or if it is influenced by language disorders. Studies have shown [3]-[5] that aphasic patients are more likely to present mathematical difficulties, particularly in tests involving numerical transcoding, like reading aloud arabic numbers and writing arabic numbers from an oral dictation. Other studies correlating language and calculation have been performed [3] [6] [7] to investigate whether numerical processing and calculation and language processes are dependent or independent activities. Some studies suggest that numerical and language processings are partially overlapping skills; for some numerical tasks, calculating and linguistic abilities are closely related, and for others, there is no correlation, with some calculation tasks being completed by patients with severe language impairment. These studies were performed involving brain-injured individuals with different types of aphasia, educational levels and professions [3] [5] [6] [8]-[10] and accounted for the variability of each individual and neurological impairment. The pattern of errors for a large sample of aphasic patients was analyzed [9] and the most severe impairment in calculating ability was found in global aphasics [11]. Patients with Broca's and Wernicke's aphasia performed similarly in quantitative terms, while patients with amnestic aphasia exhibited fewer difficulties in making calculations. The authors concluded that although the findings suggested associations between impairments in language processing and numerical tasks, one should be cautious in drawing conclusions regarding the verbal basis of general numerical skills [12].

Many observed numerical processing problems may also arise from other, nonlinguistic impairments frequently found in left hemispheric patients, including attentional deficits, short-term memory problems, or difficulties in monitoring complex sequences. Double dissociations between calculation and language abilities were also observed. The first aspect of dissociation, intact language functions and impaired calculation, has been described [13]. A case study clearly demonstrated the second aspect of dissociation, good calculation abilities despite severe language problems, where the patient correctly performed simple addition, subtraction, and multiplication and performed multidigit operations without problems [14]. Seemingly, this patient neither relied on verbal forms in any operations nor compensated for impaired verbal skills with nonverbal ones. Although this evidence supports the functional independence of language and some numerical skills, the authors nevertheless assumed that linguistic functions mediate other numerical abilities (e.g., counting, writing numerals) or preferentially support them (e.g., multiplication tables). They thus assumed that the systematic association of linguistic and numerical deficits may be informative and contribute to our understanding of the numerical difficulties observed in different clinical populations.

Despite a large number of case reports, it is important to conduct studies with groups of patients to verify whether associations and/or dissociations between language and calculation truly exist while also considering cultural and educational variability [15] [16].

The battery EC301, which was used in this study for evaluation calculation, was developed in 1994 by Delouche et al. to the adult assessment with involvement of the calculation and processing numerical after brain injury. This battery includes three systems answers to numbers: arabic digit, spelling and oral.

Thus, considering all the findings described above, this study aimed to verify a possible relationship between numerical processing and calculation difficulties and language disorders in aphasic patients.

\section{Materials and Methods}

The study was conducted in the outpatient clinic of disturbances acquired neurological speech and ginguagem and other outpatient clinics of the Speech Therapy Department at UNIFESP, São Paulo, Brazil. The sample size was calculated according to the number of patients seen at the clinic, that met the sample inclusion criteria. 
To analyze the specific difficulties encountered in numerical processing and calculation tasks among the aphasic group, we first compared aphasic and healthy adults in specific calculating tasks. Then, just were selected for this study the calculation tasks that were badly performed by aphasic patients. Then, the performance of the aphasic patients in calculation tasks was correlated to their performance in language tasks.

Control Group: Forty-four volunteers were selected (74\% female). The average education duration was $8.5 \pm$ 4.1 years, and the average age was $40.6 \pm 16.0$ years. Aphasic Group: Thirty-two patients ( $37 \%$ female) who had suffered a single left hemisphere stroke were evaluated. The mean age of the patients was $51.4 \pm 13.7$ years, and the mean education duration was $8.0 \pm 5.2$ years.

The general inclusion criteria were as follows: no history of alcoholism or drug use; no use of psychotropic medications, except for atypical neuroleptics; and the absence of visual or auditory impairments that might affect the outcome of the tests. The control group consisted of individuals who were accompanying patients, family members or friends.

This study included patients who had suffered a single left hemisphere stroke, while illiterate patients were excluded. Patients that presented motor difficulties that prevented them from performing the tasks were excluded from this study. All patients selected for the study were right-handed. All subjects were evaluated by a neurologist and underwent magnetic resonance imaging of the brain, with the following results: of the patients, $10(32 \%)$ had a lesion in the left parietal region, 9 (28\%) in the frontal-temporal-parietal, 3 (9.4\%) in the left temporal, 6 (18.5\%) in the left frontal-temporal, 3 (9.4\%) in the left temporal-parietal-occipital, and $1(3.15 \%)$ in the left parietal-occipital. Among the patients with aphasia, 16 (50\%) had anomic aphasia, 3 (9.4\%) had conduction aphasia, 4 (12.5\%) had Broca's aphasia, 1 (3.15\%) had transcortical sensory aphasia, 5 (16\%) had mist aphasia, and 3 (9.4\%) had global aphasia.

The data analyzed in the current study were collected in accordance with the Research Ethics Committee of UNIFESP, protocol No. 0346/04. All patients signed informed consent forms prior to participation.

All patients underwent an evaluation of their calculation skills using the EC301 calculation battery [17]. This battery is referred to be the most used to assess calculation abilities in adults [18] and contains 13 different tasks. Each task is domain specific and is made up of more than one subtask. Thirty-one is the total of subtasks. It was not necessary to adapt the number of stimuli of EC301 battery.

1) Counting (3 subtasks, C1, C2, C3). The subject must use different codes (phonological, Arabic, and orthographic) to produce a somewhat automatic sequence of numbers, backwards and forwards, according to different ratios (by ones, by threes, by tens).

2) Dot Counting (5 subtasks, C4, C5, C6, C7, C8). This task evaluates the capacity to compute the cardinality of a set of discrete elements (dots) with different spatial arrangements. The subject is required to point to the dots while counting aloud.

3) Transcoding (7 subtasks, C9, C10, C11, C12, C13, C14, C15). These subtasks correspond to the six possible transcodings between phonological, Arabic, and orthographic codes and number repetition. Items were selected to make their lexical-syntactical structure directly comparable from one subtask to another.

4) Arithmetic Signs (2 subtasks, C16, C17). The subject is required to name the arithmetic signs and write them as dictated.

5) Number Comparison (2 subtasks, C18, C19). The subject is required to point to the greater of two numbers, presented in Arabic (8 items) or orthographic forms (8 items).

6) Mental Computation (2 subtasks, C20, C21). This task evaluates mental calculation, requiring the subject both to access number fact knowledge and perform simple operations.

7) Estimating the Result of an Operation (1 subtask, C22). Subjects must point to the best approximation (among 4 alternatives) of the correct result of a complex operation.

8) Number Positioning on an Analog Scale (2 subtasks, C23, C24). A vertical line graded from 0 to 100 is shown to the subject, who must place a spoken or written Arabic number on the line, choosing among three possible positions.

9) Writing Down an Operation (1 subtask, C25). The subject is requested to copy a pair of two- or three-digit numbers, placing them in the conventional way for the written operation, corresponding to a given arithmetic sign.

10) Written Calculation (3 subtasks, C26, C27, C28). This task tests the subject's ability to perform addition, subtraction, and multiplication, which involves accessing numerical facts and calculation procedures such as carrying and borrowing. 
11) Perceptive Estimation of Quantity (1 subtask, C29). Subjects must estimate the weight, length, or number of objects shown in a picture.

12) Contextual Magnitude Judgments (1 subtask, C30). Given a specific and contextual situation, the subject is asked to give an interpretation of numerical size (i.e., a classroom with nine children, is the number of children too low, average, or too high?).

13) Numerical Knowledge (1 subtask, C31). This task contains questions related to numerical knowledge of specific facts, such as the number of days in a week.

Each item in EC301 was assigned a value of 2 points for a correct response and 0 points for an incorrect response. However, in a few tasks, 1 point could be awarded (i.e., if the patient gives the correct response after requesting item repetition). All subjects completed the battery tests.

In addition to the EC301 battery [4], subjects with aphasia were submitted to a language test to assess their degree of language impairment. The language evaluation used the Montreal Toulouse Protocol-Brazilian version [19] and in this study, we analyzed the performance of aphasic patients on six tests: oral and written comprehension, in which subjects would point at representations of words and simple and complex phrases, verbal and written ordering, repeating and reading words and short and long phrases and picture naming.

\section{Statistical Analysis}

In Table 2, we calculated the Spearman coefficients to verify the relationship between performance on language tasks in aphasic patients and performance on tests of EC301 battery, and p $<0.005$ was considered to indicate statistical significance, according to Bonferroni correction. $\mathrm{n}=32$.

All analyses were calculated using the statistical package SPSS (Statistical Package for Social Sciences).

\section{Results}

There was no statistically significant difference between the control and aphasic groups when comparing their years of schooling $(8.5 \pm 4.1$ versus $8.0 \pm 5.2$ years, $95 \% \mathrm{CI}=-1.7$ to 2.6 , $\mathrm{t}(74)=0.439, \mathrm{p}=0.662)$. However, the control group was significantly younger than the aphasic group ( $40.6 \pm 16.0$ versus $51.4 \pm 13.7$ years, $95 \%$ $\mathrm{CI}=-17.7$ to $-3.7, \mathrm{t}(74)=-3.06, \mathrm{p}=0.003)$ and had a higher proportion of women $\left(74 \%\right.$ versus $26 \%, \mathrm{X}^{2}=$ $8.90, \mathrm{P}=0.003)$.

The performance of patients with aphasia was significantly worse than that of the control group on subtests C1, C2, C3, C9, C10, C11, C12, C13, C14, C15, C16, C17, C19, C20, C21, C22, C26, C27, C28, C29, and C30 from the EC301 battery test, data previously reported (De Luccia, Ortiz, 2014).

Table 2 correlates the findings of the EC301 battery with the language results of the aphasic subjects.

The performance of patients with aphasia was significantly worse than that of the control group on subtests C1, C2, C3, C9, C10, C11, C12,C13, C14, C15, C16, C17, C19, C20, C21, C22, C26, C27, C28, C29, and C30 from the EC301 battery test, data previously reported (De Luccia, Ortiz, 2014).

\section{Discussion}

We observed that aphasic individuals showed changes in numerical processing and calculation tasks that were not observed in the healthy population. Although the groups were different according to age and gender, we do not believe that this difference interfered with the results, since there were no elderly subjects in our sample and there is no evidence of cognitive changes in adults [20]. As far as gender is concerned, there is a report of a little advantage of males [4] but in most studies, including one that was done with the EC 301 battery, no statistically significant difference between the performance of males and females was found [21] [22], Table 1.

The most important finding of this study is the result that aphasic individuals showed changes in numerical processing and calculation tasks that were positively correlated with linguistic task performance.

Concomitant deficits in language and calculation processing proved evident, as discussed below.

The latest research attempting to correlate language processing with numerical processing and calculation has not yet been able to clearly demonstrate an association between these two cognition domains. Questions persist regarding how language skills relate to numerical processing and calculation.

We found strong associations between oral and written comprehension tests and all calculation tests, as shown in Table 2. Table 2 shows the correlations between language and calculation tests. In general, the results of the 
Table 1. Demographic characteristics between the control group and the aphasic group.

\begin{tabular}{ccc} 
& Control & Aphasic \\
\hline Subjects & 44 & 32 \\
Age & $40.6 \pm 16.0$ & $51.4 \pm 13.7$ \\
Schooling & $8.5 \pm 4.1$ & $8.0 \pm 5.2$ \\
\hline & Female & Male \\
\hline Gender & 56 & 20
\end{tabular}

Age and schooling-values expressed as mean/years and DS.

Table 2. Correlation between performance on the EC301 battery and language tests for aphasic patients.

\begin{tabular}{|c|c|c|c|c|c|c|c|c|c|}
\hline & Test 1 & Test 3 & Test 4 & Test 5 & Test 6 & Test 7 & Test 10 & Test 11 & Test 12 \\
\hline \multicolumn{10}{|c|}{ Oral comprehension } \\
\hline $\mathrm{r}$ & 0.73 & 0.68 & 0.67 & 0.52 & 0.64 & 0.46 & 0.55 & 0.57 & 0.73 \\
\hline $\mathrm{p}$ & $<0.001^{*}$ & $<0.001^{*}$ & $<0.001^{*}$ & $0.003^{*}$ & $0.000^{*}$ & $0.008^{*}$ & $0.001^{*}$ & $0.001^{*}$ & $<0.001^{*}$ \\
\hline \multicolumn{10}{|c|}{ Repetition } \\
\hline $\mathrm{r}$ & 0.81 & 0.87 & 0.84 & 0.76 & 0.81 & 0.56 & 0.68 & 0.57 & 0.72 \\
\hline $\mathrm{p}$ & $<0.001^{*}$ & $<0.001^{*}$ & $<0.001^{*}$ & $<0.001^{*}$ & $<0.001^{*}$ & $0.001^{*}$ & $<0.001^{*}$ & $0.001^{*}$ & $<0.001^{*}$ \\
\hline \multicolumn{10}{|c|}{ Reading } \\
\hline $\mathrm{r}$ & 0.59 & 0.72 & 0.57 & 0.44 & 0.68 & 0.48 & 0.53 & 0.54 & 0.35 \\
\hline $\mathrm{p}$ & $<0.001^{*}$ & $<0.001^{*}$ & $0.001^{*}$ & 0.012 & $<0.001^{*}$ & $0.006^{*}$ & $0.002^{*}$ & $0.001^{*}$ & 0.051 \\
\hline \multicolumn{10}{|c|}{ Graphic comprehension } \\
\hline $\mathrm{r}$ & 0.81 & 0.86 & 0.86 & 0.73 & 0.87 & 0.75 & 0.78 & 0.68 & 0.63 \\
\hline $\mathrm{p}$ & $<0.001^{*}$ & $<0.001^{*}$ & $<0.001^{*}$ & $<0.001^{*}$ & $<0.001^{*}$ & $<0.001^{*}$ & $<0.001^{*}$ & $<0.001^{*}$ & $<0.001^{*}$ \\
\hline \multicolumn{10}{|c|}{ Naming } \\
\hline $\mathrm{r}$ & 0.74 & 0.79 & 0.72 & 0.47 & 0.76 & 0.50 & 0.55 & 0.62 & 0.59 \\
\hline $\mathrm{p}$ & $<0.001^{*}$ & $<0.001^{*}$ & $<0.001^{*}$ & 0.006 & $<0.001^{*}$ & $0003^{*}$ & $0.001^{*}$ & $<0.001^{*}$ & $<0.001^{*}$ \\
\hline \multicolumn{10}{|c|}{ Dictation } \\
\hline $\mathrm{r}$ & 0.86 & 0.91 & 0.78 & 0.63 & 0.83 & 0.51 & 0.75 & 0.63 & 0.56 \\
\hline $\mathrm{p}$ & $<0.001^{*}$ & $<0.001^{*}$ & $<0.001^{*}$ & $<0.001^{*}$ & $<0.001^{*}$ & $0.003^{*}$ & $<0.001^{*}$ & $<0.001^{*}$ & $0.001^{*}$ \\
\hline
\end{tabular}

p $<0.005$ indicates statistical significance after Bonferroni correction. $\mathrm{n}=32$; : Counting (C1, C2, C3); 3: Transcoding (C9, C10, C11, C12, C13, C14, C15); 4: Arithmetic Signs (C16, C17); 5: Number Comparison (C18, C19); 6: Mental Computation (C20, C21); 7: Estimation of the Result of an Operation (C22); 10: Written Calculation (C26, C27, C28); 11: Perceptive Estimation of Quantity (C29); 12: Contextual Magnitude Judgment (C30).

EC301 battery tests, on which aphasic patients performed significantly worse than the control subjects, were strongly associated with the language test results.

A association between oral comprehension, oral assessment, written comprehension, and written computation tests, based on tests with sentences and arithmetic sums, has been found in patients with lesions in the left cerebral hemisphere [23]. From these findings, these authors hypothesized that written and oral language comprehension may be involved in written and oral calculation performance, respectively. For example, when an individual fails the oral comprehension test and has difficulty pointing at the correct figure after a verbal or written command like "show where the girl drinks water", he also fails to perform the oral calculation "thirteen minus 
eight" or the written calculation "13 - 8".

In this work, strong correlations were found. We can observe (Table 2) that oral comprehension was strongly correlated with the counting, transcoding, arithmetic sign, and contextual magnitude judgment tests. Written comprehension and repetition tests also correlated strongly with all calculation tests. We observed a strong correlation between reading and counting and transcoding and mental computation tests. Comparing the naming and calculation tests, we observed strong correlations with the counting, transcoding, arithmetic signs, mental computation, perceptive quantity estimation, and contextual magnitude judgment tests. For dictation, the strongest correlations were found with counting, transcoding, arithmetic signs, number comparison, mental computation, written calculation, and perceptive quantity estimation tests.

The only test that showed no correlation with the oral comprehension test was test 7-estimated operation result. However, we observed that in both the aphasic and control groups, the estimated result test (C22) was the most difficult. We can assume that the test is already mathematically complex for this study group, i.e., the performance may not be specifically related to not understanding the task. A association between the language tests evaluated in this study and some EC301 battery tests was observed. We can consider that the direct or indirect involvement of skills such as oral counting, alphabetic or orthographical number writing, oral and written comprehension, and repetition is necessary for tests 1 -numeric sequence, 3 -transcoding, 4 - sign recognition, and 6-mental calculation.

In accordance with the international literature, strong correlations were observed between oral and written comprehension tests and oral and written calculation (Table 2), suggesting that oral and written linguistic comprehension interferes in understanding the calculation to be performed. The inability, due to linguistic changes in aphasia, to understand task instructions or to give a verbal or written response may thus lead to an interdependency between language and numerical processing and calculation.

We believe that these correlations may be present due to the reduction of language processing resources and that oral comprehension may interfere in the oral calculation test (C20), the magnitude judgment test (C29), and the estimating result and quantity test (C30), (Table 2). The best example of the possible changes in oral calculation tests (C20) was the difficulty encountered by aphasics in performing even simple multiplication and subtraction calculations. We believe that these difficulties arose due to gaps in lexical access, both for numerical memory when executing the calculation and for arithmetical rules themselves. Both failure in the phonological loop and the inability to recall prior knowledge may have generated difficulties relating to "multiplication tables". In contrast, the magnitude judgment and result estimation tests (C29 and C30) may suggest more complex changes from the comprehension perspective, as they evaluate a change in the context and/or semantic judgment interpretation. This would imply failure of the quantifier, the role of which is to quantify observations and experiences, translating them into numbers by counting and measuring and thus being a base for mathematics and language [24] [25].

In the aphasic group, arithmetic rule memory was also correlated with the language tests (Table 2). These changes can be explained by lexical-semantic failures, based on symbol names and meanings. When analyzing aphasic patient performance, we found that most patients recognized the numbers but had difficulty recognizing or naming some arithmetic signs $(+,-, \times,=)$. Furthermore, we observed that many individuals performed calculations orally when the evaluator mentioned the sign. We believe that this failure could also be attributed to difficulty in graphically recognizing each symbol. Although able to visually recognize symbols, the aphasic cannot establish relationships between them because the graphical symbol is merely a meaningless pictorial representation for these subjects. The difficulty of asymbolic acalculia suggests that arithmetic signs are symbols of a different semiotic system in comparison to written language and numbers and can thus be considered an ideographic note, as each sign has a restricted and independent value [26]. Individuals who had difficulties naming and writing arithmetic signs (i.e., addition, multiplication, division, and equal signs) were also more likely to have the possible diagnosis of asymbolic acalculia (in some cases) and lexical-semantic disruption (in others).

Although some evidence, mainly in single case reports, has upheld the notion of the functional independence of language and number processing and calculation, we believe that some tasks, such as those involving counting, transcoding, and mental and written calculations, depend on linguistic processing. Counting serially requires learned numerical concepts [3]. Numerical transcoding abilities depend on a central component that performs all transcoding and calculations [10]. For numerical comprehension or calculations, mechanisms are necessary to translate numerical inputs, whether in orthographic, arabic, or verbal form. Moreover, numerical production mechanisms have implications that require translation and abstract representations of the appropriate forms of 
output for each notation system (verbal, orthographic, or arabic). To complete this process, a distinction must also be made between lexical and syntactic numerical processing, in which a series of verbal, orthographic, and arabic transformations in numeric comprehension are performed.

During the transcoding tests in this study, lexical and syntactical errors were observed in most cases, and misspellings and omissions were observed for all types of aphasia. According to the results found for tasks involving language and arithmetic skills, such as transcoding tests, a relationship was observed between language and mathematics.

The semantic models proposed by McCloskey, 1992, suggest that transcoding produces a semantic representation of numerical processing. For this to occur there must be a full abstract internal representation, which provides information for the arabic, verbal, and orthographical representations of each number, which allows for transcodification from one code to another. The numerical input would be transformed into abstract internal representations, and the output would be converted into another code. Considering the many skills involved in this process, we might expect that patients with brain injuries would encounter major difficulties in these tasks, as such relationships seem stronger in apparently similar skills, including transcoding between different representations of numbers and graphophonetic decoding. This raises questions about the status of the numerical transcoding ability bearing in mind that its mathematical components are the concept of numbers and a sense of the positional value of digits (i.e., ones, tens, hundreds, and thousands). This ability also involves essentially linguistic components, including phonological and syntactic aspects [27]. From this perspective, the numerical transcoding ability is central to the discussion regarding the relationship between language and mathematics, as it seems to be a skill that combines mathematical and linguistic components. The involvement of linguistic and mathematical components suggests the importance of further investigating the specific nature of the numerical transcoding task to observe not only its connections with strictly linguistic skills, such as graphophonetic decoding.

Written calculations were more difficult for aphasic patients. Changes in understanding and lexical and written difficulties, both commonly found in aphasics, may also affect performance on these tasks [28]. In this regard, the systematic association of linguistic and numerical deficits may be informative and contribute to our understanding of numerical difficulties in different clinical populations. The acalculia pattern found in the aphasia group can be explained as follows: both calculation routes may have been damaged, leading to deficiencies in tasks that require semantic number comparison.

This study has some limitations: despite the fact that $50 \%$ of our sample comprised anomic aphasics, the remaining was heterogeneous. This did not allow us to perform an analyses considering aphasia type/severity. Also, duration of being aphasic in the aphasic group they were not raised.

Further investigation could consider the severity of language and calculation disorders in specific groups of aphasic patients.

\section{Conclusions}

Despite that our findings show correlations between losses language and numerical processing and calculation, it is not possible to state as a whole that the calculation is directly related to language. The individual variability in processing linguistic or numerical information and calculation must be considered. Furthermore, the hypothesis that the language and calculation systems are independent, but interdependent in its activity is the most accepted in this study.

As we consider the aphasic in the general population, as was done in this study, terms evidence that the language skills of listening and graphics, repetition, reading and writing are directly related to the difficulties found evidence of oral and graphic calculation and transcoding evidence who are involved reading assignments, writing and repetition, suggesting that linguistic processing is involved in the performance of various mathematical tasks.

\section{References}

[1] Ardila, A., Galeano, L.M. and Rosselli, M. (1998) Toward a Model of Neuropsychological Activity. Neuropsychology Review, 8, 171-190. http://dx.doi.org/10.1023/A:1021618218943

[2] Nieder, A. and Dehaene, S. (2009) Representation of Numbers in the Brain. Annual Review of Neuroscience, 32, 185208. http://dx.doi.org/10.1146/annurev.neuro.051508.135550

[3] Basso, A., Burgio, F. and Caporali, A. (2000) Acalculia, Aphasia and Spatial Disorders in Left and Right Brain- 
Damaged Patients. Cortex, 36, 265-280. http://dx.doi.org/10.1016/S0010-9452(08)70528-8

[4] Dellatolas, G., Deloche, G. and Salinas, D. (2001) Assessment of Calculation and Number Processing Using the EC301 Battery: Cross-Cultural Normative Data and Application to Left-and-Right Brain Damage Patients. Journal of the International Neuropsychological Society, 7, 840-859.

[5] Ardila, A. and Rosselli, M. (2002) Acalculia and Dyscalculia. Neuropsychology Review, 12, 179-231. http://dx.doi.org/10.1023/A:1021343508573

[6] Grafman, J., Kampen, D., Rosenberg, J., Salazar, A.M. and Boller, F. (1989) The Progressive Breakdown of Number Processing and Calculation Ability: A Case Study. Cortex, 25, 121-133. http://dx.doi.org/10.1016/S0010-9452(89)80012-7

[7] Grana, A., Hofer, R. and Semenza, C. (2006) Acalculia from a Right Hemisphere Lesion Dealing with "Where” in Multiplication Procedures. Neuropsychologia, 44, 2972-2986. http://dx.doi.org/10.1016/j.neuropsychologia.2006.06.027

[8] Klessinger, N., Szczerbinski, M. and Varley, R. (2007) Algebra in a Man with Severe Aphasia. Neuropsychologia, 45, 1642-1648. http://dx.doi.org/10.1016/j.neuropsychologia.2007.01.005

[9] McCloskey, M. (1992) Cognitive Mechanisms in Numerical Processing: Evidence from Acquired Dyscalculia. Cognition, 44, 107-157. http://dx.doi.org/10.1016/0010-0277(92)90052-J

[10] Delazer, M., Girelli, L., Semenza, C. and Denes, G. (1999) Numerical Skills and Aphasia. Journal of the International Neuropsychological Society, 5, 213-221. http://dx.doi.org/10.1017/S1355617799533043

[11] Dahmen, W., Hartje, W., Bussing, A. and Sturm, W. (1982) Disorders of Calculation in Aphasic Patients Spatial and Verbal Components. Neurpsychologia, 20, 145-153. http://dx.doi.org/10.1016/0028-3932(82)90004-5

[12] Warrington, E.K. (1982) The Fractionation of Arithmetical Skills: A Single Case Study. The Quarterly Journal of Experimental Psychology, 34, 31-51. http://dx.doi.org/10.1080/14640748208400856

[13] Rossor, M.N., Warrington, E.K. and Cipolotti, L. (1995) The Isolation of Calculation Skills. Journal of Neurology, 242, 78-81. http://dx.doi.org/10.1007/BF00887820

[14] Domahs, F., Moeller, K., Huber, S., Willmes, K. and Nuerk, H.-C. (2010) Embodied Numerosity: Implicit Hand-Based Representations Influence Symbolic Number Processing across Cultures. Cognition, 116, 251-266. http://dx.doi.org/10.1016/j.cognition.2010.05.007

[15] Cappelletti, M., Butterworth, B. and Kopelman, M. (2012) Numeracy Skills in Patients with Degenerative Disorders and Focal Brain Lesions: A Neuropsychological Investigation. Neuropsychology, 26, 1-19.

[16] Deloche, G. and Seron, X. (1982) A Differential Analysis of Skills in Transcoding Quantities between Patients with Broca's and Wernicke's Aphasia. Brain, 105, 719-733. http://dx.doi.org/10.1093/brain/105.4.719

[17] Deloche, S., Seron, X., Larroque, C., Magnien, C., Metz-Lutz, M.N., Riva, I., Schils, J.P., Dordain, M., Ferrand, I., Baeta, E., Basso, A., Cipolotti, L., Salinas, C.D., Howard, D., Gaillard, F., Goldenberg, G., Mazzucchi, A., Stachowiak, F., Tzavaras, A., Vendrell, J., Bergego, C. and Pradat-Diehl, P. (1994) Calculation and Number Processing: Assessment Battery; Role of Demographic Factors. Journal of Clinical and Experimental Neuropsychology, 16, 195208. http://dx.doi.org/10.1080/01688639408402631

[18] Delazer, M., Girelli, L., Granà, A. and Domahs, F. (2003) Number Processing and Calculation-Normative Data from Healthy Adults. The Clinical Neuropsychologist, 17, 331-350. http://dx.doi.org/10.1076/clin.17.3.331.18092

[19] Parente, M.A.M.P., Ortiz, K.Z., Soares, E.C.S., Scherer, L.C., Fonseca, R.P., Joanette, Y., Lecours, A.R. and Nespoulous, J.-L. (In Press) Bateria Montreal-Toulouse de Avaliação da Linguagem—Bateria MTL-BR. Vetor Editora, São Paulo.

[20] De Luccia, G.P. and Ortiz, K.Z. (2014) Ability of Aphasic Individuals to Perform Numerical Processing and Calculation Tasks. Arquivos de Neuro-Psiquiatria, 72.

[21] Deloche, G., Souza, L., Braga, L.W. and Dellatolas, G. (1999) A Calculation and Number Processing Battery for Clinical Application in Illiterates and Semi-Literates. Cortex, 35, 503-521. http://dx.doi.org/10.1016/S0010-9452(08)70815-3

[22] De Luccia, G.P. and Ortiz, K.Z. (2009) Performance of Brazilian Population on EC301 Battery: A Pilot Study. Arquivos de Neuro-Psiquiatria, 67, 432-438. http://dx.doi.org/10.1590/S0004-282X2009000300012

[23] Baldo, J.V. and Dronkers, N.F. (2007) Neural Correlates of Arithmetic and Language Comprehension: A Common Substrate? Neuropsychologia, 45, 229-235. http://dx.doi.org/10.1016/j.neuropsychologia.2006.07.014

[24] Kobuchi, M.P. (2007) Floating Numerals and Floating Quantifiers. Lingua, 117, 814-831. http://dx.doi.org/10.1016/j.lingua.2006.03.008

[25] Liang, J., Yin, J., Chen, T., Chen, H., Ding, X. and Shen, M. (2012) Number Representation Is Influenced by 
Numerical Processing Level: An ERP Study. Experimental Brain Research, 218, 27-39. http://dx.doi.org/10.1007/s00221-012-2998-7

[26] Ferro, J.M. and Botelho, M.A. (1980) Alexia for Arithmetical Signs. A Cause of Disturbed Calculation. Cortex, 16, 175-180. http://dx.doi.org/10.1016/S0010-9452(80)80032-3

[27] Deloche, G. and Seron, X. (1982) From One to 1: An Analysis of Transcoding Process by Means of Neuropsychological Data. Cognition, 12, 119-149. http://dx.doi.org/10.1016/0010-0277(82)90009-9

[28] Baldo, J.V. and Dronkers, N.F. (2007) Neural Correlates of Arithmetic and Language Comprehension: A Common Substrate? Neuropsychologia, 45, 229-235. http://dx.doi.org/10.1016/j.neuropsychologia.2006.07.014 